\title{
Experimental results of tomographic reconstruction on ONERA laboratory MCAO bench
}

\author{
Anne Costille ${ }^{1, a}$, Cyril Petit ${ }^{1}$, Jean-Marc Conan ${ }^{1}$, Caroline Kulcsár ${ }^{2}$, and Henri-François Raynaud ${ }^{2}$ \\ 1 ONERA - DOTA Unité HRA \\ 2 L2TI Université Paris 13
}

\begin{abstract}
Classic Adaptive Optics (AO) is now a proven technique to correct turbulence for earth based astronomical telescopes. But the corrected Field of View (FoV) is however limited by the anisoplanatism effect. Many concepts of wide field AO systems are now under study, especially for ELT's instruments. These systems allow a tomographic reconstruction of the turbulent volume thanks to the use of several WaveFront Sensors (WFS). They can provide for instance a correction in a specific direction (Laser Tomography AO system) with one Deformable Mirror (DM) or in a wide FoV (Multi Conjugate AO system) with several DMs conjugated to different altitudes. The performance optimization of such complex systems raises inevitably new questions in terms of calibration and control. We present our current developments on performance optimization of wide field AO system. We show that performance can be significantly improved with tomographic control based on Linear Quadratic Gaussian control, compared to more standard methods. The associated calibration and system modeling strategy is described. The results presented here are the first experimental validations of LQG control in closed-loop MCAO and LTAO configurations. These tests have been performed on HOMER bench, recently developed at ONERA and devoted to MCAO and wide field AO laboratory research. This bench implements two deformable mirrors and a wide field Shack-Hartmann WFS.
\end{abstract}

\section{Introduction}

Classic adaptive optics (AO) [1] is now a proven technique to correct turbulence on earth based astronomical telescopes. The corrected Field of View (FoV) is however limited by the anisoplanatism effect. To increase the FoV corrected, new concepts of AO, called hereafter Wide Field AO (WFAO), are now under development, especially for ELT's instruments. These concepts use several Guide Stars (GSs) to analyze the turbulent volume and then provide a tomographic reconstruction of atmospheric turbulence. The correction can be applied with one DM to correct a specific direction (Laser Tomography AO (LTAO) [2]) or with several DMs conjugated in altitude to correct a wide FoV (MultiConjugate AO (MCAO) [3]). These new concepts require the development of new control and calibration strategies. It is also important to develop new laboratory benches, devoted to WFAO, to test these approaches.

We present in this article the results of our experimental study of WFAO concepts, performed with the bench HOMER, developed at ONERA. First we quickly describe HOMER bench and its main characteristics to present the experimental conditions (section 2). We describe our control approach based on a Linear Quadratic Gaussian (LQG) control that has been used to perform a tomographic reconstruction in real-time in closed-loop conditions (section 2.1). We also present the calibration strategy established during our tests (section 2.2). The last part of this article is devoted to the experimental results obtained on HOMER in LTAO and MCAO using LQG control (section 3).

\section{HOMER bench}

HOMER bench, presented in fig. 1 - left, is a WFAO laboratory bench in visible wavelengths. It is devoted to implement and validate new concepts of WFAO. It allows to study calibration issues:

\footnotetext{
a e-mail: anne.costille@onera.fr
} 

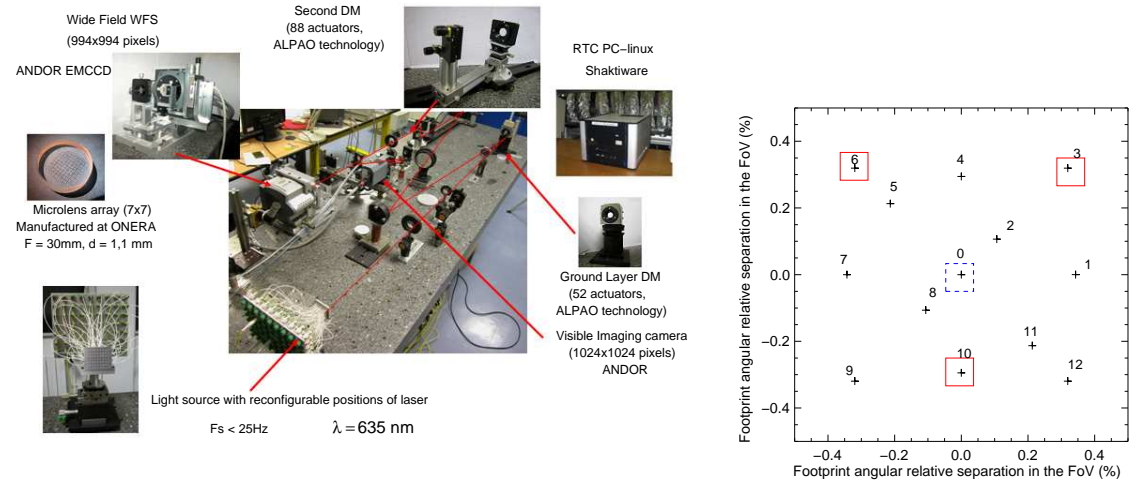

Fig. 1. Left: HOMER bench and its main components. Right: GSs configurations (solid boxes for WFAO) and science star constellation Star 0 surrounded by a dashed blue box corresponds to the direction of correction in LTAO.

impact and correction of field aberrations, calibration of models for control implementation including development of identification process of these models... It is also dedicated to control laws and WFS algorithms implementation and comparison. HOMER bench has a reconfigurable multi-source system composed of fiber coupled laser diodes in the visible wavelengths $(\lambda=635 \mathrm{~nm})$, associated to a collimator. All GSs and science stars in the FoV are natural ones. It also has a turbulent space where phase screens could be placed to generate the turbulence. Two DMs, developed by ALPAO, allow to correct the turbulence. One is conjugated with the pupil and the other with a given altitude. For the time being we use the DMs themselves to generate the turbulence. This configuration allows to work in a well known environment and presents interesting features for control validation. A single wide field Shack-Hartmann WFS analyzes the light coming from several GSs. Multi directional analysis is then performed through numerical windowing. It allows to change easily the number and configuration of the GSs, while reducing the calibration and synchronization issues. A wide field imaging camera is used for performance estimation. The WFAO loops are controlled by a Real Time Computer (RTC), manufactured by Shaktiware, working at $10 \mathrm{~Hz}$. The RTC allows to implement different kinds of control laws, and in particular LQG control [4] described in section 2.1. More details about the bench can be found in $[5,6]$.

The pattern of science targets and GSs positions in the FoV is presented on fig. 1 - right. Star number 0 is in the center of the FoV and stars 3, 6 and 10 are used for WFS in all WFAO results presented in the paper. We identify a given star in the FoV by its angular position with respect to star 0 . The position can also be expressed in relative separation $\delta$ at a given altitude $h: \delta=\alpha h / D$, where $\alpha$ is the angular position of the star and $D$ the pupil diameter. The altitude DM is conjugated to an altitude that ensures $\delta= \pm 0.34$ for stars number 3, 6, 9 and 12. By analogy, if we consider a telescope a telescope with $D=8 \mathrm{~m}$ and two stars separated by 1 arcmin, a footprint relative separation of 0.34 is obtained for a turbulent layer at 9347 meters. We project Kolmogorov phase screens on the DMs to generate the turbulence. We scale the turbulence so as to obtain an equivalent strength in each layer $\left(C_{n}^{2}=50 \%\right.$ in each layer), a global $D / r_{0}$ equals to 7 at $635 \mathrm{~nm}$ and $V / D=1 \mathrm{~Hz}$ for each turbulent layer, where $V$ is the wind speed in the layer.

\subsection{LQG control formalism}

The work presented in this paper is based on the use of LQG control for WFAO. We recall in this part the formalism of this control law, that has already been fully described especially in [6,7]. We first introduce the formalism and the modeling of WFAO concepts. Figure 2 describes the general WFAO 


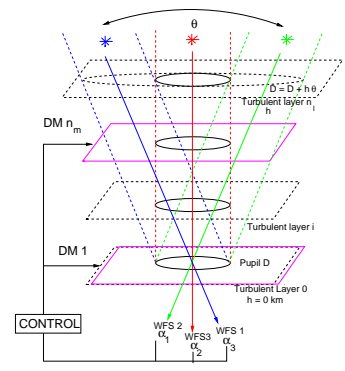

Fig. 2. Principle of a WFAO system. Turbulence is composed of $\ell$ layers and analyzed in $n_{g s}$ directions thanks to $n_{g s}$ WFSs conjugated with the GSs. Correction is performed by $m$ DMs, conjugated at $m$ altitudes.

configuration using $m$ DMs for correction and $n_{g s}$ for analysis. We consider a two frame delay and the DMs dynamics is neglected. All the variables are considered in discrete-time [8].

The telescope's diameter is denoted by $D$ and it is assumed that the atmosphere can be modeled by $\ell$ discrete turbulent layers statistically independent. Each layer $i$ is characterized by its altitude $h_{i}$. Considering a maximal FoV $\theta, \varphi_{i, n}^{t u r}$ is the average turbulent phase on interval $[(n-1) T, n T]$ in the layer $i$, corresponding to the discrete instant $n$. It is defined over a meta-pupil delimited by the FoV, the diameter of which is $D_{i}=D+h_{i} * \theta$. The turbulence can be expanded on a convenient modal basis (Zernike basis...).

A turbulent phase in the volume $\varphi_{n}$ is defined by the concatenation of the turbulent phases of each layer $i$. Considering several analysis directions $\alpha$, the turbulent phase seen in the telescope pupil is equal to $\phi_{\alpha, n}^{t u r}=\mathbf{M}_{\alpha}^{\ell} \varphi_{n}^{\text {tur }}$, where $\mathbf{M}_{\alpha}^{\ell}$ is a linear operator performing the sum of all contributions of each turbulent layer $\ell$ in all directions $\alpha$. The correction phase in the directions $\beta$ in the pupil is then given in a similar way by $\phi_{\beta, n}^{\text {cor }}=\mathbf{M}_{\beta}^{m} \varphi_{n}^{\text {cor }}$.

We consider a WFAO system composed of $m$ DMs conjugated at several altitudes and let $\mathbf{u}_{n}$ be the voltage vector at instant $n$ gathering each DM voltage vectors $\mathbf{u}_{j, n}(j \in\{1, \ldots, m\})$ then $\mathbf{u}_{n}=$ $\left(\left(\mathbf{u}_{1, n}\right)^{\mathrm{t}},\left(\mathbf{u}_{2, n}\right)^{\mathrm{t}}, \ldots,\left(\mathbf{u}_{m, n}\right)^{\mathrm{t}}\right)^{\mathrm{t}}$. We note $\mathbf{N}$ the global influence matrix for all the DMs. This is then a bloc diagonal matrix, each bloc $j$ of the diagonal containing the influence matrix $\mathbf{N}_{j}$ of the $j^{\text {th }} \mathrm{DM}, j \in$ $\{1, \ldots, m\}$. The correction phase in the volume $\varphi_{n}^{\text {cor }}$ is then defined by $\varphi_{n}^{\text {cor }}=\mathbf{N u}_{n-1}$.

So, considering $n_{g s}$ GSs and directions of analysis $\alpha$ and $n_{o b j}$ objects of interests with directions of interest $\beta$, the residual wave front measurements obtained in directions $\alpha$ are:

$$
\mathbf{y}_{n}=\mathbf{D}_{n_{g s}}\left(\mathbf{M}_{\alpha}^{\ell} \varphi_{n-1}^{t u r}-\mathbf{M}_{\alpha}^{m} \mathbf{N u}_{n-2}\right)+\mathbf{w}_{n},
$$

where $\mathbf{D}_{n_{q s}}$ contains $n_{g s}$ row repetition of matrix $\mathbf{D}$, which describes the linear response of a WFS, and assuming that all WFSs are identical. The measurement noise $\mathbf{w}_{n}$ is a generalized measurement noise vector for all WFSs.

The residual phase in directions of interest $\beta$ is given by:

$$
\phi_{n-1}^{r e s}=\mathbf{M}_{\beta}^{\ell} \varphi_{n-1}^{\text {tur }}-\mathbf{M}_{\beta}^{m} \mathbf{N u}_{n-2} .
$$

The WFAO optimality criterion is obtained as the generalization of the AO criterion [8] which aims at minimizing the residual phase variance in a given FoV:

$$
\mathbf{J}(\mathbf{u})^{\text {mcao }}=\lim _{n \rightarrow+\infty} \frac{1}{n} \sum_{j=1}^{n}\left\|\mathbf{M}_{\beta}^{\ell} \varphi_{j}^{\text {tur }}-\mathbf{M}_{\beta}^{m} \mathbf{N u}_{j-1}\right\|^{2} .
$$

The control problem in equation (3) finds its solution in a two step resolution. This classic result is known as the stochastic separation theorem $[9,10]$ and states that the optimal control $\mathbf{u}_{n}$ can be constructed by separately solving a deterministic control problem and a stochastic minimum variance estimation problem. The stochastic minimum variance estimation problem consists in estimating/predicting the turbulent phase. Its solution is provided, in the linear Gaussian case, by the Kalman 
filter ${ }^{1}$, based on the state-space representation of the system. As turbulence is generated by the DMs in the current experiment, a natural way is to represent the turbulence phase in the DM space. We assume that the turbulent phase corresponds to $\varphi_{n}^{u}=\mathbf{N}^{t u r} \mathbf{v}_{n}$ where $\mathbf{N}^{t u r}$ is a block-diagonal matrix containing the influence matrices of both DMs used to generate the turbulence, and $\mathbf{v}_{n}$ corresponds to the turbulent voltages applied on the DMs. For a simple turbulent phase model of the form $\varphi_{n+1}^{u}=\mathbf{A}^{u} \varphi_{n}^{u}+v_{n}$, a state-space model can be defined as in [6]. All the matrices of the state-model being stationary, the asymptotic formulation of the Kalman filter is applied. The estimate $\hat{\varphi}_{n / n}^{u}$ of the turbulent phase $\varphi_{n}^{u}$ and the prediction of the turbulence $\hat{\varphi}_{n+1 / n}^{u}$ can be found in [6]. The LQG optimal control comes down to the following and compact form:

$$
\begin{gathered}
\hat{\varphi}_{n+1 / n}^{u}=\mathbf{A}^{u} \hat{\varphi}_{n / n}^{u}=\mathbf{A}^{u} \hat{\varphi}_{n / n-1}^{u}+\mathbf{A}^{u} \mathbf{H}_{\infty}\left(\mathbf{y}_{n}-\mathbf{D} \mathbf{M}_{\alpha}^{\ell} \hat{\varphi}_{n-1 / n-1}^{u}\right), \\
\mathbf{u}_{n}=\mathbf{P} \hat{\varphi}_{n+1 / n}^{u} .
\end{gathered}
$$

where $\mathbf{P}=\left(\left(\mathbf{M}_{\beta}^{m} \mathbf{N}\right)^{\mathrm{t}} \mathbf{M}_{\beta}^{m} \mathbf{N}\right)^{\dagger}\left(\mathbf{M}_{\beta}^{m} \mathbf{N}\right)^{\mathrm{t}} \mathbf{M}_{\beta}^{\ell}$ is the projection of the phase estimated in the turbulent layer and in the FoV in the directions of interest and onto the DMs, and $\mathbf{H}_{\infty}$ is the asymptotic Kalman gain given in [6]. The description of the LQG control formalism shows that this control is based on the use of models of the turbulence (matrix $\mathbf{A}^{u}$ ) and the system (matrices $\mathbf{D}, \mathbf{N}, \mathbf{M}_{\alpha}^{\ell} \ldots$ ) that have to be identified.

\subsection{Calibration strategy for WFAO tests}

Before presenting the experimental results obtained in WFAO, we propose to describe our calibration strategy used on HOMER to implement LQG control and to test WFAO concept. First we have evaluated the optical imaging path quality of HOMER bench. We have noticed that the bench is affected by Non Common Path Aberrations (NCPA) in the FoV that degrade the SR without turbulence. We have not corrected them during our tests. Thus, after optimization of the optical alignment, we have obtained a mean SR of $56 \%$ in the FoV with a standard deviation of $5.8 \%$. We can notice that NCPA are quite limited in the FoV in our case.

Another step of the calibration of HOMER bench has consisted in evaluating the correct position of the WFS windows, that point the GS. This would correspond to the relative pointing directions of the WFSs on a multi-WFS system. Our first strategy has been to calculate the position of the WFS windows thanks to the knowledge of the system parameters. We have observed that the object formed by the GSs is distorted by the optical path and then the position of the WFS windows does not correspond to the theoretical ones. This slope bias or distortion, after propagation through the tomographic process, can not be properly corrected by the DMs configuration on HOMER, leading to a loss of performance. To avoid this impact on the performance, we have corrected the position of the WFS windows in order to not correct the distortion [11].

We have shown in section 2.1 that LQG control relies on models of the turbulence and of the system. The calibration of these models is essential to ensure the best performance of LQG control. In our case where the turbulence is generated by the DMs, the calibration is simplified. First we have established the model of the turbulence based on the matrix $\mathbf{A}^{u}$. This matrix is time-constant and diagonal. In the DM basis, one can even take a matrix proportional to identity since turbulent voltages exhibit a similar correlation time for all actuators. We then calculate the temporal correlation for each turbulent voltage $\mathbf{v}$ and identify the best fit to the short term correlation at one step. The second part of the calibration concerns the identification of the models of the system. These models can be evaluated from numerical models of the components or directly from experimental data. Another approach, that has not been considered in our test, consists in using experimental data to identify key parameters of the components and then to create models with these parameters [12]. In our case where the DMs generate the turbulence, all models of the system can be represented by interaction matrices between the WFSs and the DMs used for the correction or the generation of the turbulence. A study of the numerical

\footnotetext{
1 If the Gaussian assumption is released, the Kalman filter provides the best linear unbiased estimate
} 
models has shown that we have a good representation of the model of $\mathbf{D}$ and $\mathbf{N}$ but the models of $\mathbf{M}_{\alpha}^{\ell}$, $\mathbf{M}_{\beta}^{m}, \ldots$ present some errors. To limit models errors, we have thus chosen to use interaction matrices obtained experimentally by push-pull of each actuator of the DMs. A first study of the behavior of LQG control in presence of model errors has shown that the control can be affected by model errors if they are very important. We have also noticed that it is always better to use a consistent set of matrices (numerical or experimental ones) to establish the models of the control, even if the matrices present models errors.

\section{Experimental results obtained on HOMER bench}

We present in this section experimental results obtained in WFAO with HOMER bench. First, we have performed LTAO tests in closed-loop. The turbulence is thus analyzed in three directions (stars 3, 6 and 10 on figure 1-right) and the correction is applied with one DM conjugated to the entrance pupil. The goal of LTAO is to provide a high correction in one specific direction of the FoV, where no bright GS can be used for $\mathrm{AO}$ analysis and correction. We choose to correct in the direction of star 0 in the center of the FoV. The interest of using LQG control is great in this case because it performs a reconstruction and a prediction of the turbulent volume in real time. It then allows to project the turbulence in a specific direction along which no measurements are available to obtain optimal DM correction. Table 1 gathers the results obtained in LTAO for these stars, compared with the performance obtained in AO in the same turbulent conditions when the GS corresponds to star 0. In LTAO, a SR of 55\% is reached on star 0 , which is close to the performance of $60 \%$ of SR in AO. The difference is due to the residual tomographic error. The correction deteriorates in the FoV in the same manner that in a classic on-axis $\mathrm{AO}$, due to anisoplanatism. LQG control provides a very significant correction on axis in closed-loop in LTAO and allows to perform a tomographic estimation of the turbulence.

We also have tested the performance of the LQG control in MCAO and compared the results obtained with a standard integrator control providing a least-square reconstruction. In MCAO, we use three GSs (stars 3, 6 and 10) and we use both DMs for correction. The integrator control is obtained with the truncated generalized inverse of the generalized interaction matrix between the WFSs and the DMs. As we are in good signal-to-noise ratio conditions, we use a global integrator gain of 0.5 and filter 20 modes to obtain the best performance. Note that this control law is entirely dependent on the directions of analysis and optimization of the correction in other directions is hardly possible. The goal of MCAO is to provide a good and uniform correction in the whole FoV. As the integrator implicitly reconstructs the phase in the DM space, the results obtained in MCAO with an integrator control are very good: we obtained 53\% of SR averaged in the FoV. LQG control gives a better performance with a higher SR on all measured directions of interest and in average with $56 \%$ of SR in the FoV. Table 2 presents the results obtained with both controls. These results give a successful demonstration of the applicability of LQG control on a real MCAO system despite inevitable uncertainties on system models and experimental conditions. The cases presented here validate the LQG controlled MCAO and also LTAO configuration, with the advantage of a structurally stable control law. Note that we have compared all experimental results with numerical results, obtained by an end-to-end simulation of HOMER bench. The experimental results are confirmed numerically, after taking into account the impact of the non common path aberrations in the numerical results. Numerical simulations presented in [7] have shown the greatest interest to implement LQG control in a realistic turbulence case when turbulence is not generated by the DMs: the performance is better than with integrator and more homogeneous in the FoV. Simulations of HOMER case when the turbulence is generated by phase screens have also given the same behavior.

\section{Conclusion}

We have presented in this article tests of WFAO concepts (MCAO and LTAO) using LQG control. We first have described the bench HOMER, used for these tests, and the main equations of LQG control that allows to perform a tomographic reconstruction of the turbulence and then a correction in 
First conference on Adaptive Optics for Extremely Large Telescopes

Table 1. SR obtained on different stars in classical AO in presence of turbulence and in LTAO with LQG control.

\begin{tabular}{lllll}
\hline Star number & 0 & 3 & 6 & 10 \\
\hline Experimental SR in AO (\%) & 60 & 13 & 12 & 20 \\
\hline Experimental SR in TAO (\%) (LQG) & 55 & 12 & 10 & 18 \\
\hline
\end{tabular}

Table 2. Statistics of the SR obtained in MCAO with integrator and LQG control.

\begin{tabular}{llll}
\hline & Mean SR (\%) & Maximum SR (\%) & Minimum SR (\%) \\
\hline MCAO with integrator control & 53 & 62 & 39 \\
\hline MCAO with LQG control & 56 & 66 & 40 \\
\hline
\end{tabular}

a specified FoV. We also have studied calibration aspects for WFAO concepts, in particular concerning the identification of the models for LQG control. The calibration procedure, especially concerning models identification, is going to be enriched with methods that minimize a quadratic criterion to evaluate the models from experimental data [12].

The implementation of LQG control in WFAO has been applied to test LTAO and MCAO configurations on HOMER in closed-loop. Our study has shown the feasibility of LQG control to reconstruct the turbulent volume and to apply a specific correction. In particular, we have realized the first experimental validation of closed-loop LTAO, that provides a high correction in a specific direction where there is no bright star. We have also performed the first experimental validation of LQG in closed-loop LTAO and MCAO. The results obtained in MCAO are most encouraging for implementation of LQG control for future systems.

Developments of HOMER bench are ongoing to simulate even more realistic WFAO systems, leading to a useful tool for ELT instruments studies. In particular, we plan to implement and compare other sub-optimal controls. More complex effects such as mis-conjugation between turbulence and DM layers, or Laser GSs effects need to be addressed as well.

\section{References}

1. F. Roddier, Adaptive Optics in astronomy (Cambridge University Press, 1999).

2. N. Hubin, R. Arsenault, R. Conzelmann, B. Delabre, M. Le Louarn, S. Stroebele and R. Stuik, Comptes Rendus de l'Académie des Sciences Physique 6, (2005) 1099-1109

3. J.-M. Beckers, Proceedings of a ESO Conference on Very Large Telescopes and their Instrumentation, (ESO Conference and Workshop Proceedings, 1988), No. 30, 693-703.

4. B. Le Roux, J.-M. Conan, C. Kulcsár, H.-F. Raynaud, L. M. Mugnier and T. Fusco, J. Opt. Soc. Am. A 21, (2004) 1261-1276

5. A. Costille, C. Petit, J.-M. Conan, T. Fusco, C. Kulcsár and H.-F. Raynaud, Proceedings Soc. Photo-Opt. Instrum. Eng., (2008) paper 7015-152

6. A. Costille, C. Petit, J.-M. Conan, C. Kulcsár, H.-F. Raynaud and T. Fusco, (submitted to J. Opt. Soc. Am. A )

7. C. Petit, J.-M. Conan, C. Kulcsár and H.-F. Raynaud, J. Opt. Soc. Am. A 26, (2009) 1307-1325

8. C. Kulcsár, H.-F. Raynaud, C. Petit, J.-M. Conan and P. Viaris de Lesegno, Opt. Express 14, (2006) 7464-7476

9. P. Joseph and J. Tou, AIEE Trans. on Appl. and Indus 80, (1961) 193-196

10. P. Whittle, Optimization over time - Dynamic programming and stochastic control (John Wiley \& sons, 1982).

11. A. Costille, Les optiques adaptatives grand champ : stratégie de correction et validations expérimentales $(\mathrm{PhD}$. Thesis, 2009).

12. F. Rigaut, A. Garcia, B. Neichel, M. Bec and M. Boccas, in this conference 